\title{
COMPLEJOS SIMBÓLICOS DE ANTEO Y ATLAS EN LA POESÍA DE SEAMUS HEANEY ${ }^{1}$
}

\author{
Juan Ráez Padilla
}

\begin{abstract}
Resumen: En este artículo abordamos el estudio de la ambivalencia simbólica de la tierra en la poesía de Seamus Heaney, y más concretamente en su poemario The Spirit Level (1996). Se analizan, de una parte, los valores símbolos de índole positiva dentro del que denominamos complejo simbólico de Anteo. De otra, constatamos el valor negativo de la sobrecarga que no pocas veces caracteriza a la tierra en el denominado complejo simbólico de Atlas. A través de ambos opuestos / complementarios nuestro artículo incidirá en la dialéctica, tensión y movimiento propios de la poética heaneyiana.
\end{abstract}

Palabras clave: Seamus Heaney, simbología, tierra, agua, tensión, equilibrio, movimiento.

\begin{abstract}
This article focuses on the symbolic ambivalence of earth in the poetry of Seamus Heaney, especially in his collection The Spirit Level (1996). On the one hand, it analyses positive symbolic values within the so-called symbolic complex of Antaeus; on the other hand, the author explores in his symbolic complex of Atlas the negative value of overload which often characterizes Heaney's earth. Through these complementary opposites, the article emphasizes such Heaneyian poetic features as dialectics, tension and movement.
\end{abstract}

Key words: Seamus Heaney, symbolism, earth, water, tension, balance, movement.

En la introducción a su libro Seamus Heaney: Searches for Answers (2003), Eugene O’Brien enfatiza en la poética del norirlandés conceptos tales como dualidad, alteridad, o interacción, desembocando todos ellos en la premisa de que su poesía aspira a expresar (2003: 3) "la complejidad de relaciones inter-subjetivas" (el énfasis es nuestro). ${ }^{3}$ Sus versos, por tanto, son un complejo en el que confluyen la voz individual y la voz colectiva, la afirmación y la duda, el pasado y el futuro. Todas estas interconexiones, pensamos, se materializan con frecuencia en la interacción simbólica de los elementos naturales y las imaginerías que les son anejas. En este mismo sentido, y como superación de las frecuentes trabas restrictivas en relación con la pertenencia a una comunidad dada, el propio O'Brien (2003: 4) se refiere a la implementación por parte de Heaney de “enunciaciones simbólicas mucho más amplias de identidad individual y cultural" (el énfasis es nuestro). La identidad, aduce este crítico, no viene impuesta para el poeta, sino que éste atiende a la pluralidad de estructuraciones sociales y culturales, construyendo a partir de ellas una identidad propia.

\footnotetext{
$1 \quad$ Fecha de recepción: abril 2007.

Fecha de aceptación y versión definitiva: julio 2007.

2 Profesor Ayudante Doctor, Departamento de Filología Inglesa, Universidad de Jaén; $\square$ jraez@ujaen.es.

3 Cuando no se especifica lo contrario, las traducciones al español de fuentes en lengua inglesa son nuestras.
} 
Ésta, asimismo (2003: 4-5), "ha de ser creada a través del lenguaje y la imaginería" (el énfasis es nuestro), aduce O’Brien. El símbolo, el lenguaje mismo, pues, son las armas con las que cuenta el poeta para trasladar lo complejo real a lo complejo poético. De ahí -pensamos- la patente similitud entre la tensión poética en Heaney y la tensión en el contexto socio-político de Irlanda del Norte. La identidad nacional o personal, pues, pueden manifestarse como un estado mental ${ }^{4}$ (O'Brien 2003: 4) que trasciende el inmovilismo, cambiante y dinámico como el propio símbolo, accesible a través de aquellas enunciaciones simbólicas que transcriben entre velos un poeta, una comunidad, un país.

En las páginas que siguen intentaremos arrojar alguna nueva luz sobre esta dinámica simbólica, reveladora de la complejidad que, como O'Brien, destacamos en la obra de Seamus Heaney. Incidiendo en la llamativa ambivalencia simbólica heaneyiana, centraremos aquí nuestra atención en algunos valores y antivalores simbólicos que la tierra muestra en el poemario The Spirit Level (1996). Siguiendo con nuestro anterior argumento sobre la complejidad heaneyiana, englobaremos estos dos tipos de valores opuestos-complementarios en sendos complejos simbólicos. Los valores, es decir, aquéllos que son expuestos desde una perspectiva positiva, pertenecerán a lo que denominaremos complejo simbólico de Anteo; los antivalores, es decir, aquellos rasgos simbólicos que el poeta presenta de una u otra manera como negativos, se inscribirán dentro del complejo simbólico de Atlas... Ambos héroes mitológicos, como veremos, no son ajenos a la obra de Heaney. El primero de ellos recibía su inquebrantable fuerza a través del permanente contacto con la tierra, por lo que el complejo simbólico que lleva su nombre acapara el valor regenerativo del elemento terreno, su virtud, su riqueza, su positividad en la más amplia gama de valores. Por su parte, Atlas fue condenado a cargar el mundo sobre sus espaldas, de ahí que las realidades simbólicas que asociemos a su nombre implicarán el peligro y la pesadumbre de la sobrecarga terrena, la negativa limitación del inmovilismo resultante. En cierta manera, el complejo de Atlas representa un exceso del complejo de Anteo, incurriendo así en el desequilibrio. Ambos complejos suscitarán, por tanto, el motivo del equilibrio simbólico, tan característico de la poesía del norirlandés. The Spirit Level (1996) contiene no pocas manifestaciones de esta ambivalencia, representativa, a su vez, del movimiento, otro rasgo fundamental de la poética de Seamus Heaney.

La palabra y la tierra están íntimamente relacionadas en la poesía de Seamus Heaney. "Digging", recordemos, es el título del primer poema de la primera colección de Seamus Heaney, Death of a Naturalist (1966): "Between my finger and my thumb / The squat pen rests. / I'll dig with it" (1966: 4, vv. 29-31). Sobre este poema escribe el norirlandés en "Feeling into Words", definiendo la poesía como "a dig, a dig for finds that end up being plants" (1980: 41). Cabe destacar, por tanto, su temprana y sempiterna devoción por la imaginería rural. Ésta es precisamente -por poner un ejemplo- el cauce de expresión del movimiento

\footnotetext{
4 O’Brien retoma aquí un término utilizado por el propio Heaney en "Squarings" xxxvii: "In famous poems by the sage Han Shan, / Cold Mountain is a place that can also mean / A state of mind. Or different states of mind / At different times" (1991: 97, vv. 1-4). Recordemos, asimismo, que el título de otro libro de O'Brien es Seamus Heaney: Creating Irelands of the Mind (2002).
} 
versal en "Poet's Chair" 2. Heaney se retrotrae a los años de infancia, espectador de la labor del arado realizada por su padre. Sentado en el centro del campo, el poeta expresa que aquella vivencia constituía, de alguna manera, una forma de presciencia, foreknowledge, (1996: 47, 3, vv. 6-7): "Of the poem as a ploughshare that turns time / Up and over"5 (el énfasis es nuestro). El poeta como labrador: así como la hoja del arado voltea la tierra, el verso poético, aduce Heaney, remueve el tiempo. Para la analogía se hace especialmente reveladora una apreciación que Heaney hace en su prosa crítica, referente a la coincidencia etimológica que, muy a propósito de la simbología poético-agraria, vincula verso y arado, escritor y labriego. Verso proviene del latín versus, que significaba una línea de un poema -tal y como hoy en día se utiliza el vocablo-, aunque también designaba el giro que el labrador da al terminar un surco al final de un campo y disponerse a empezar otro (1980: 65). De hecho, éste es su sentido original: mientras que la línea de la prosa proseguía en disposición continua, el poema -más entrecortado-se asemejaba formalmente a un campo de surcos que versan, giran de sentido. Teniendo en cuenta esta temprana epifanía lingüística para el norirlandés, la distancia entre la labor de su padre y la suya propia se hará mucho más corta. Mucho más íntima la conexión entre aquel recuerdo de infancia y la labor del verso en la edad adulta.

Otra imagen del padre en "A Call" manifiesta el afecto y la ternura del campesino para con la tierra. El poeta hace una llamada de teléfono. Después de hablar con su madre, ésta va a buscar a su esposo, que se encuentra desherbando fuera de la casa. En la espera Heaney casi puede ver, sentir, la minuciosidad del cuidado paternal de la tierra:

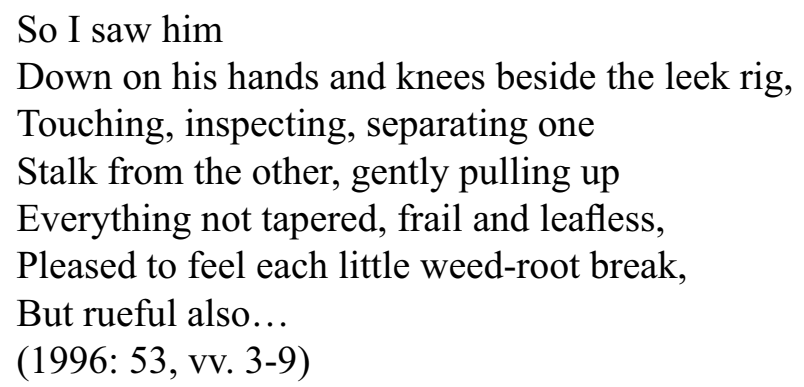

El contacto humano-terreno es aquí íntimo, estrecho. El mimo y la pulcritud empleados convierten aquella escena casi en una tarea artística. En un cuadro rupestre que Heaney contempla con emoción. Como en el poema anterior, la labor campesina adquiere cualidad poética. El labrador se complace en aquel acto de purificación terrena. No obstante, su amor a todo vástago de la tierra es tal que hasta el arrepentimiento (rueful) se deriva de la escarda de las malas hierbas. Este sentimiento agridulce causado por la separación de la tierra es una constante en la poesía de Seamus Heaney: el movimiento ascendente libera, independiza -en este caso, purga, sanea la tierra-; por otro lado, la pérdida de la raíz no puede concebirse en su poesía -independientemente del valor aéreo- sin cierto grado de compunción y pesadumbre. Algo se rompe dentro del padre, dentro del poeta, al oír el crujir de aquellas frágiles hierbas. El tic-tac del reloj de pared retumba en el silencio de la espera telefónica. De repente, el poeta cae en la cuenta: "if it were nowadays, / This is how Death

La aliteración entre ploughshare y poem refuerza la identificación entre ambos. 
would summon Everyman" (1996: 53, v. 14). La melancolía se apodera de la escena... La conciencia de nuestra propia fragilidad terrena. El viejecito, finalmente, se pone al aparato: "Next thing he spoke and I nearly said I loved him" (1996: 53, v. 15).

Otra figura que manifiesta la dimensión artística de la tierra, más en concreto de la piedra, es "An Architect". Heaney le caracteriza como una persona exigente, misteriosa, absorta en su trabajo. La tierra es también su adoración, "minding the boulder / And the raked zen gravel" (1996: 58, vv. 2-3). Como en "The Gravel Walks", piedra (cal y pizarra azul) es todo a lo que se reduce la verdad del arte del arquitecto (el énfasis es nuestro): "Talking his way back into sites and truths / The art required and his life came down to: / Blue slate and whitewash, [...]" (1996: 58, vv. 10-15).

Con frecuencia la tierra y el agua interactúan en el complejo simbólico de Anteo, de manera que la simbología de la pasta -tomando prestadas las palabras de Gaston Bachelard- se configura como una de las manifestaciones poéticas más veneradas. La tierra de donde el verso de Heaney toma su mayor brío no es tanto rocosa o árida como legamosa, arcillosa. En "To a Dutch Potter in Ireland", poema dedicado a la ceramista holandesa Sonja Landweer, encontramos el primer exponente en el volumen de este tipo de simbología. El poema comienza con la evocación de una tierra de la infancia que se muestra sucia, "slabbery, clabbery, wintry, puddled" (1996: 2, 1, v. 3), dentro de la cual el poeta finalmente encuentra, bajo capas de humus, el tesoro terreno de la arcilla del río Bann, en Irlanda del Norte. Contrastan en los versos de Heaney la tierra estancada de agua (nótese el valor peyorativo de la adjetivación en la cita de arriba) con la brillosa arcilla, mezcla ecuánime de tierra y agua, protomateria para la creación artística tanto del poeta (Heaney) como de la alfarera (Landweer). ${ }^{6}$ La propia lengua inglesa, curiosamente, los vincula en la proximidad fónica entre poet (poeta / poetisa) y potter (alfarero/a). No en vano, a lo largo del poema Heaney establece una cómplice relación con la ceramista, imaginando una infancia en común donde podrían haber llevado a cabo "the small forbidden things - / Worked at mud-pies or gone too high on swings, / Played 'secrets' in the hedge or 'touching tongues", (1996: 3, 1, vv. 19-21). El lodo es materia de inspiración en la edad adulta como lo es en la infancia, plastilina de la naturaleza con la que temprano se juega a crear formas, estirando aquí, reduciendo allá, creando pasteles (mud-pies) o cualquier otro objeto como por arte de magia. ${ }^{7}$ La arcilla de Bann es, pues, símbolo del pre-conocimiento artístico, del comienzo, de la inocencia. En un poema posterior Heaney (1996: 5) escribirá, precisamente, sobre la presciencia contenida en la tierra: "earth's foreknowledge gathered in the earth" (el énfasis es nuestro). Oculta en un pequeño hoyo, la arcilla es rescatada por la psique del poeta adulto como aquellos recuerdos imaginados de la infancia, manchados y sepultados, al igual que aquélla, entre frías y entarquinadas capas de violencia fratricida en Irlanda del

\footnotetext{
“Todos los grandes soñadores terrestres”, escribe Bachelard, "aman a la tierra así, veneran a la arcilla como materia del ser" (1994: 149).

7 Según Bachelard, el modelado de la pasta es un ensueño simbólico que nos retrotrae a la infancia, situada “en el límite de dos universos: el universo del agua y el universo de la tierra” (1994: 91): “¡Modelado! ¡Sueño de infancia, sueño que nos devuelve a nuestra infancia!". Ya en la edad adulta, "hay un modo de recobrar esas posiblidades perdidas [...]. Ese modo es la literatura. [...] Pluma en mano -con sólo que queramos ser sinceros- recobraremos todos los poderes de la juventud, los revivimos tal como eran, en su ingenua confianza, con sus alegrías rápidas, esquemáticas y seguras" (1994: 110). The Spirit Level (1996) abunda precisamente en esta filosofía bachelardiana sobre la recuperación literaria de la infancia.
} 
Norte. También en los Países Bajos, durante la Segunda Guerra Mundial, de manera que aquel columpio o los pasteles de barro no quedan más que en un sueño poético compartido, perdido: "Night after night instead, in the Netherlands, / You watched the bombers kill" (1996: 3, 1, vv. 23-24). En este contexto de suciedad física y simbólica, la arcilla de Bann constituye la "diatomita verdadera" (2000: 25, v. 6), "the earth's old ointment box" (1996: 2,1, v. 9), en alusión a los ungüentos y especias frescas llevadas al Santo Sepulcro para la unción del cuerpo de Jesucristo (Collins 1997: 339). Cura, creación, conocimiento, valores todos ellos contenidos en el credo terreno de Anteo.

Con respecto al complejo simbólico de Anteo y la devoción heaneyiana por la tierra, "The Gravel Walks" es quizás el poema más significativo dentro de la colección. El propio título nos revela ya que dos son las ideas principales: por un lado -como a lo largo de todo el poemario-, el movimiento (walk); por otro, el símbolo terreno de la grava. Los motores del mundo se ponen en funcionamiento, el remolino interior del río talla en su seno el pedernal y la arenisca (1996: 39, vv. 5-8) -nótese el valor catalítico del movimiento, de vital importancia en el poemario-. La escena se asemeja en gran medida a la escena bíblica de la creación del mundo. No obstante, la naturaleza se presenta casi como una fábrica natural, contexto metafórico que anticipa la posterior usurpación humana de este tesoro de la naturaleza: "An eternity that ended once a tractor / Dropped its link-box in the gravel bed / And cement mixers began to come to life" (1996, 39, vv. 11-13). La grava es sustituida por la manipulación humana para la invención del cemento, símbolo de la industria y el progreso humanos. La chispa terrena por percusión natural entre los pequeños cantos rodados se intensifica hasta el delirio ígneo de la construcción artificial, "as if / The Pharaoh's brickyards burned inside their heads" (1996: 39, vv. 15-16). Se trata de una realidad dual a la que Heaney no fue ajeno desde la infancia. A medio camino entre el conflicto y la paz, entre los libros y la azada, no son pocas las veces que su poesía discurre por la encrucijada entre el hombre y la naturaleza, la contemplación y la acción, la modernidad y la tradición. Como ocurría en "Terminus", dios romano y protector de los límites de los campos. En este poema también se yuxtapone la bellota al tornillo -cuando se buscaba por entre el suelo-, la chimenea de una fábrica a la montaña -cuando el joven poeta levantaba la mirada al cielo-, el ruido del motor del tren al del galope de un caballo (1987: 4, vv. 1-6). Son obvias aquí las implicaciones del mencionado valor del equilibrio (hombre-naturaleza, tierra-cielo) en la escritura del irlandés. Ante la dicotomía grava-cemento de "The Gravel Walks" Heaney entona el credo de Anteo, e invoca:

Hoard and praise the verity of gravel.

Gems for the undeluded. Milt of earth.

Its plain, champing song against the shovel

Soundtests and sandblasts words like 'honest worth'.

(1996: 39, vv. 17-20)

La grava es riqueza, verdad. Se encuentra dentro de cada uno de nosotros (1996: 39, v. 22). Con ella el poeta declara buscar la "absolución del cuerpo" (2000: 111, v. 27). Muchos son los poemas en los que Heaney busca una redención poética. Aquí parece encontrarse en el tesoro de la grava, de la cual el poeta se acopia y amontona -piénsese en las conno- 
taciones de adoración del verbo (to) hoard - con el mismo fervor que los hombres voltean la mezcladora para producir cemento (1996: 39, v. 15): "Mixed concrete, loaded, wheeled, turned, wheeled" (nótese el efecto enfático del asíndeton). La codicia del cemento acabó con aquella eternidad del mundo a la que el poeta se refiere en el verso 11 . La avidez en el acopio de grava, de igual manera, "kept you slow and steady" (1996: 40, v. 25), te hace esclavo -escribe Heaney- de una pesada carretilla que nos encorva y mortifica los huesos (1996: 40, vv. 26, 28). El poema constituye, así pues, un compendio práctico del valor regenerativo de la tierra, así como, por otro lado, de su inmovilismo. Una oda a la imperturbable zancada de Anteo; una elegía a Atlas, pilastra petrificada.

Ya en un poema de Wintering Out Heaney (1972: 15) se define como "Dives, / hoarder of common ground" (el énfasis es nuestro). El principio simbólico es el mismo que en "The Gravel Walks": de un lado, el poeta se muestra orgulloso del acopio y posesión de la tierra común, "the four green fields" (Frazier 2001: 10), en oposición a otros campos perdidos (1972: 13) y extraños (1972: 17). La tierra es también aquí riqueza simbólica, especialmente a favor de la configuración de una identidad nacional. No obstante, como también señala Frazier (2001: 10), Heaney ofrece señales de que tal celosa posesión terrena es equivocada, pues lo convierte en un arquetipo del pecado, Dives (en latín, hombre rico) o Epulón, quien negó su ayuda a Lázaro y fue por ello condenado (Lucas 16.19-31). ${ }^{8}$

Atlas, dios portador de la tierra, es una figura clásica presente, de forma explícita o implícita, en buena parte de la poesía de Seamus Heaney. Es manifiesto el atractivo que ofrece al irlandés este gigante de la tradición helénica, dejándose sentir su huella en la simbología terrena de la sobrecarga y el estancamiento. Aparece de manera explícita, por ejemplo, en "Anything Can Happen", traducción que Heaney hace de la oda 1, 34 de Horacio:" "Ground gives. The heaven's weight / Lifts up off Atlas like a kettle lid" (2004: 11, vv. 13-14). En el original, escalofrío humano ante el trueno de Júpiter y el temblor de la terra tremens (en oposición a la terra firma), no aparece más que una referencia al Atlántico. No obstante, el poeta declara haber tomado la libertad de añadir una nueva estrofa -así como suprimir la primera de la versión latina- tomando como base a Atlas (2004: 19). Ello da muestra de la relevancia de tal figura en la poética heaneyiana, presente ya en "Antaeus", un temprano poema de 1966 sobre la lucha mito-simbólica entre Anteo (tierra) y Hércules (aire): "Let each new hero come / Seeking the golden apples and Atlas. / He must wrestle with me before [...]" (1975: 3, vv. 13-15). En The Spirit Level (1996), asimismo, constituye una presencia mítico-simbólica destacada. Aparece de forma explícita en "Mycenae Lookout", como homónimo sobre el que el centinela de Micenas proyecta el peso de su remordimiento: ${ }^{10}$

\footnotetext{
8 Estrictamente hablando, cabe mencionar que este hombre rico al que Frazier alude no tiene vinculación alguna con la tierra. Sí la tiene, por el contrario, el otro hombre rico descrito en Lucas 12.16-21.

9 Esta traducción fue escrita por Seamus Heaney tras los ataques terroristas sufridos por la ciudad de Nueva York el 11 de septiembre de 2001, y publicada por vez primera en The Irish Times el 17 de noviembre de aquel mismo año.

10 El centinela de "Mycenae Lookout" -narrador poético de los hechos- es testigo del adulterio y el ardid de Clitemnestra contra Agamenón, aunque, en su indecisión entre confidente de la reina y súbdito del rey, nada hace finalmente para evitar el regicidio.
} 
The ox's tons of dumb inertia stood, head-down, and motionless as a herm. Atlas, watchmen's patron, would come into my mind, the only other one up at all hours, ox-bowed under his yoke of cloud out there at the world's end.

The loft-floor where the gods and goddesses took lovers and made out endlessly successfully, those thuds and moans through the cloud cover were wholly on his shoulders. Sometimes I thought of us apotheosized to boulders. called Aphrodite's Pillars. (1996: 35, 4, vv. 19-36)

Ambos, Atlas y centinela, sostienen sobre sus espaldas el peso del adulterio. El primero, el que cometen los dioses y las diosas del Partenón. El segundo, el de Clitemnestra y Egisto. En las dos estrofas de arriba varios son los símbolos que inciden en los valores de carga e inmovilismo propios de la gravidez terrena. Sus dos protagonistas, al final de la segunda estrofa, quedan literalmente transpuestos en piedra (boulders), pilares de la diosa del amor, Afrodita. Anteriormente, de igual forma, el centinela se nos presenta tan inmóvil como una hermes (herm), pilastra cuadrangular de proporciones humanas sobre la que descansaba la cabeza de un dios, generalmente (de ahí su propio nombre), el del dios Hermes, mensajero y protector de los viajantes. De hecho, las pilastras-hermes, muy abundantes en la Grecia clásica, eran frecuentes en los caminos como postes o lindes. Aquí, por tanto, encontramos un símbolo que manifiesta la auto-inculpación en el hecho de mantenerse al margen, mudo para la denuncia y sordo en connivencia, piedra limítrofe que alarga el punzante eco de aquellas palabras sobre la vejada Casandra: "No such thing / as innocent/ bystanding" (1996: 30). Si bien el equilibrio, la neutralidad y otras formas de in-betweenness heaneyiana aspiran con frecuencia a una afirmación positiva de ecuanimidad, móvil y sin cortapisas, aquí encontramos justo lo contrario, una forma de auto-crítica por la falta de acción y movimiento, ruedas, y no pilares, sobre las que normalmente friccionan la moral y la técnica poéticas del escritor norirlandés. Otro símbolo que incide en estos mismos valores es el del buey. Conocido es su tradicional valor del trabajo, la obligación, el sacrificio (Cirlot 2001: 441), imaginería del sobrepeso que en los versos de arriba también sirve para caracterizar la carga-remordimiento del narrador. La inercia muda (dumb) del buey (cf. v. 19 ss. arriba) pesa toneladas (tons), obliga a bajar la cabeza (head-down), encorvando (ox-bowed) al centinela como a Atlas lo obliga el yugo (yoke) del mundo. El poema comienza precisamente con una cita de la obra de Esquilo que apila buey, carga y mudez a un mismo tiempo: "The ox is on my tongue" 
(1996: 29). Poco después aparece la imagen de la lengua del centinela de Micenas "Like the dropped gangplank of a cattle truck, / Trampled and rattled, running piss and muck" (1996: 29, 1, vv. 13-14). Expresiones todas ellas de una misma simbología de la coerción del remordimiento, la obligación, el sacrificio, losas que muestran la imaginería terrena en su faceta más restrictiva. El buey del poema número 1 torna toro en el poema número 4 (1996: 36, 4, vv. 49-51): "My own mind was a bull-pen / where horned King Agamemnon / had stamped his weight in gold" (el énfasis es nuestro). Las connotaciones de bull-pen son múltiples, aunque Heaney las hilvana magistralmente bajo una misma manifestación simbólica del complejo de Atlas: en primer lugar, y como acabamos de mencionar, bull se muestra paralelo a la imaginería animal con que comienza la secuencia versal (ox). En segundo lugar, considerado en su conjunto (bull-pen), el compuesto incide en la misma imaginería del aprisionamiento / carga emocional (cf. weight). ${ }^{11}$ En tercer lugar, bull-pen se muestra próximo a ballpen (bolígrafo), de manera que la voz del centinela se entrecruza con la del propio poeta que escribe. El centinela no denuncia el adulterio de la reina. No previene a su rey de la muerte que le espera en su propia casa. Los lingotes de oro que éste le ofreciera pesan demasiado en la conciencia, toril sobre el que estampa su plúmbea pezuña el cornudo (horned) Agamenón: "I moved beyond bad faith: / for his bullion bars, his bonus / was a rope-net and a blood-bath" (1996: 36, 4, vv. 55-57). La imaginería rural (ox, cattle truck, bull-pen), sempiterna en la obra del norirlandés, se entrecruza con el mito griego. Todas estas manifestaciones simbólicas, no en vano, nos hablan de la vida y obra del propio Seamus Heaney: buey en la labranza poética de la tierra; centinela micénico que se debate entre la neutralidad y el intervencionismo; Atlas sobre cuyos hombros doloridos recae la columna de la causa católico-nacionalista. ${ }^{12}$

Sacrificial es el peso que soportan las manos de san Kevin en "St Kevin and the Blackbird". Según cuenta la leyenda, mientras que el santo rezaba arrodillado y con los brazos extendidos, palmas hacia arriba, un mirlo se posó sobre una de ellas para anidar. Movido por la piedad, san Kevin permaneció inmóvil hasta que los polluelos salieron del cascarón. Ante tal acto de paciente sacrificio, Heaney se pregunta por lo que ocurre dentro del cuerpo y la mente del piadoso irlandés durante todo el tiempo que permanece quieto:

Imagine being Kevin. Which is he?

Self-forgetful or in agony all the time

From the neck on out down through his hurting fore-arms?

Are his fingers sleeping? Does he still feel his knees?

Or has the shut-eyed blank of underearth

Crept up through him? Is there distance in his head?

(1996: 20, vv. 14-19)

\footnotetext{
11 Bull-pen: "A pen or enclosure for bulls; spec. a square enclosure made of logs, freq. used in early times as a place of confinement; hence, a lock-up or prison. Also transf., any enclosure or enclosed area" (OED, 2001).

12 En este sentido de equilibrio entre lo terreno y lo aéreo, entre la experiencia norirlandesa y el mito griego, quisiéramos destacar las siguientes palabras de Osterwalder: "This interweaving of metaphors from the lowly ground of the peatbog or cowshed and the Olympian heights of the Greek classics represents Heaney's development and achievement in a nutshell” (1997: 32).
} 
Dolor consciente e indolente olvido de sí mismo son las dos opciones que Heaney imagina para san Kevin. Al final del poema el irlandés contempla la segunda de ellas, pues reflejado en el río profundo del amor -nótese la benignidad de la simbología acuática- "he has forgotten self, forgotten bird / And on the riverbank forgotten the river's name" (1996: 21, vv. 23-24). Asistimos de esta manera a un proceso simbólico de petrificación que anula mente y cuerpo, convirtiendo también a san Kevin en una pilastra, un Atlas que sostiene la vida animal con paciencia sobre-humana. Su fijeza es sacrificada columna que salvaguarda la prole del mirlo. No obstante, Heaney no centra su mirada tanto en el altruismo de su acción como en el anulador efecto de la misma sobre su persona. La dedicación y el sacrificio, no en vano, no son ajenos al poeta irlandés, que desde muy temprano encauzó su poesía por el áspero y pedregoso trazado que le imponían, de una manera u otra, las vicisitudes de la historia norirlandesa más reciente. Por más que sus brazos, como los del santo, pudieran querer abrirse -palmas arriba- a las alturas metafísicas. "St Kevin and the Blackbird" quizá sea, en este sentido, un poema de desgaste más que de heroicidad. Una advertencia sobre los riesgos de la obligación y el compromiso. Una tierna historia sobre dos brazos que quieren ser alas, pero tornan maderos de cruz. El efecto paralizante, note el lector, proviene del seno de la tierra. Sus profundidades, que en tantas ocasiones regurgitan el legamoso verbo de la musa de Heaney, contienen aquí, en cambio, una ponzoñosa gravedad que trepa (crept up) como una alimaña reptante e inyecta en los pies de san Kevin el mal de Atlas. El subsuelo inocula así el lento veneno de la abnegación petrificante en el santo irlandés, el cual queda tan inmóvil y absorto como la figura femenina en terracota descrita en "The Sharping Stone": "Her right forearm and hand held out as if / Some bird she sees in her deep inward gaze / Might be about to roost there" (1996: 60, vv. 33-35). No es casualidad, en nuestra opinión, que Heaney utilice en los dos casos vocabulario idéntico (forearm, hand held out / hold his hand, 1996: 20, v. 10), que los dos hagan las veces de árbol para un pájaro, que ambos muestren un marcado ensimismamiento impuesto por la tarea de soportar un peso. Se trata de sendas escenas poético-pictóricas -especialmente por lo inmóvil de las mismas- que revelan la misma impronta impresa sobre los hombros de Atlas. O sobre los hombros magullados del propio Heaney (1996: 49, vv. 49-51), quien en "The Swing" hace las veces del gigante heleno y se libera de la carga que le impedía alzarse por encima de ella: "we sailed / Beyond ourselves and over and above / The rafters aching in our shoulderblades" (el énfasis es nuestro).

"Weighing In" comienza con el dolor por no poder levantar una pesada carga. Se trata de una pesa de veinticinco kilos, "A solid iron / Unit of negation" (1996: 17, vv. 1-2). La tierra aparece aquí como un símbolo de anulación e inmovilismo. El simbolismo geométrico es digno de reseñar, pues tal lastre tiene una forma cuadrada (squared-off, 1996: 17, v. 4), tradicionalmente asignada a la tierra (Chevalier y Gheerbrant 1996: 347; Cirlot 2001: 98, 231). Los apetitos de la gravedad en "Kinship" (1975: 37, IV, v. 24) contrastan con esta plúmbea materialización de la misma como "Gravity's black box" (1996: 17, v. 7). Su color negro completa una caracterización heaneyiana que concuerda con la tradicional visión de la tierra como el más pesado de los elementos naturales, oscuro y de geometría cuadrangular. Se trata de la inmovilidad hecha tierra, "harmless-looking / Until you tried to lift it, then a socket-ripping, / Life-belittling force" (1996: 17, vv. 4-6). Sin embargo, la dualidad permite el movimiento y la superación del inmovilismo. El equilibrio es el cata- 
lizador del cambio: hagamos contrabalanza con otra pesa sobre una báscula-puente recién engrasada "And everything trembled, flowed with give and take" (1996: 17, v. 12). Nótese el efecto dinamizador de la imaginería acuática sobre la simbología terrena: la lubricación (freshly greased, 1996: 17, v. 11) de la báscula permite que el movimiento fluya (flowed). La dualidad, en resumidas cuentas, implementa la anulación de la carga, superando así el complejo de Atlas.

Esta imaginería del equilibrio adquiere poco después, no obstante, una dimensión mucho más sombría. El poema se mueve de lo elemental simbólico a la esfera de lo comunitario, concretamente a la reflexión sobre el conflicto norirlandés. Una voz ronca irrumpe en el diálogo poético afirmando: "Peace on earth, men of good will, all that / Holds good only as long as the balance holds" (1996: 17-18, vv. 21-22). En todo conflicto, argumenta esta voz, el sufrimiento sólo es tolerable si su peso es equivalente al sufrido en el otro lado. Al igual que en la báscula-puente, la carga se sobrelleva con la contrabalanza. El poeta que habría lanzado las piedras del silencio en "Punishment" (1975: 31, vv. 30-31) es instado ahora a hacerlo en imperativo (1996: 18, vv. 34-36): "Still, for Jesus' sake / Do me a favour, would you, just this once? / Prophesy, give scandal, cast the stone" (el énfasis es nuestro). Esta voz poética, dicho sea de paso, no es la voz personal del propio Seamus Heaney, quien ha mantenido a lo largo de su carrera un tono mucho más neutral, apolítico y reconciliador. En este sentido, el léxico empleado es revelador: la expresión for Jesus' sake resuena con el mismo tono agrio que aquella otra, for fuck's sake (1996: 25, v. 27), empleada por un republicano que, pocas páginas después en "The Flight Path" 4, importuna al poeta para pedirle una mayor implicación en la causa nacionalista (1996: 25, vv. 25-30). Conocida es de sobra, no obstante, la negativa del poeta a convertirse en profeta de tal causa. A ello le insiste esta voz poética, que utiliza hasta dos veces, también en imperativo, el verbo profetizar (1996: 18, vv. 29, 36). El poema, por tanto, apunta hacia delante en la colección. También lo hace hacia atrás: la identidad de esta corrosiva voz podría ser un álter ego del albañil de "Damson" -justo el poema anterior a "Weighing In"-, quien reprocharía a Heaney el consejo que le diera en aquel poema. En él, el escritor le insta a no emplear el filo reluciente de su pala-espada, sino a utilizar su esparavel como escudo para expulsar a los vengativos espectros de ultratumba. Aquí se lamenta de tal decisión, pues cuando un golpe certero habría sido justo, cuando el contraataque debiera haber sido llevado hasta sus últimas consecuencias,

You countered that it was my narrowness

That kept me keen, so got a first submission.

I held back when I should have drawn blood

And that way (mea culpa) lost an edge.

A deep mistaken chivalry, old friend.

At this stage only foul play cleans the slate.

(1996: 18-19, vv. 43-48)

Nótese el efecto contrabalanza (countered) del consejo poético de Heaney, el cual rehuye de posiciones simplistas y unilaterales (narrowness), como la de la violencia terro- 
rista. Ello se hace patente en el movimiento y contra-movimiento simbólicos del poema, así como en esta confluencia de voces e identidades que aquí y allá se muestran ya optimistas, ya pesimistas, agoreras. Éste es precisamente, como hemos argumentado, el caso de la oposición entre la heroicidad reconciliadora del albañil y esta otra voz retaliadora, entre la acción destructiva y el "sufrimiento pasivo" -el cual "hace girar el mundo" (2000: 61, vv. 19-20) -, entre la refulgencia del filo de su paleta constructora-fulgor inspirativo para Heaney- y aquel otro filo (edge) que, en la ecuanimidad, se pierde en la batalla (cf. cita arriba). El equilibrio y la simbología alquímica, por otro lado, aparecen instrumentalizados al servicio de la apología a la violencia. El justo medio aristotélico es aducido por el integrista como base ideológica de la retaliación: "just weighing in / Is what it must come down to, and without / Any self-exculpation or self-pity" (1996: 18, vv. 38-40). Por tanto, el equilibrio se configura como un símbolo ambivalente cuyo lado más nefasto descansa aquí sobre la demagogia y la propaganda. No en vano, la nivelación propuesta en "Weighing In" choca de frente con la dimensión ético-espiritual del proyecto material -en el sentido bachelardiano de imaginación material de los elementos- de El nivel. La alquimia también es instrumentalizada en la defensa del juego sucio (foul play, cf. cita arriba) para la supuesta limpieza moral en el conflicto (cleans the slate, cf. cita arriba), para volver a retroceder desde el campo hasta la línea de batalla, expectantes y desconfiados. El poeta trastoca así la expresión idiomática (to) wipe the slate clean, con un significado mucho más reconciliador: olvidar errores u ofensas pasadas, borrarlos de la pizarra y volver a comenzar de nuevo. En el caso del conflicto norirlandés, según esta voz ominosa, only foul play cleans the slate. Sólo la retaliación equilibrante nos permite borrar todos los palitos de tiza que, en la media pizarra de cada comunidad, recuentan el número de difuntos. Hasta que alguien vuelva a apretar un gatillo y otro suba de nuevo, para apuntar, a la palestra. Este funesto give and take (1996: 17, v. 12), este sopesar en uno y otro lado de la balanza es, desgraciadamente también, la simbología que mejor representa otros conflictos en el mundo, como el que se cobra hoy tantas víctimas en Oriente Próximo...

"Weighing In" es un poema profundamente ambivalente: el uno (la pesa) es inmóvil; el dos permite el movimiento y la interacción. Toda una filosofía de enorme potencialidad poética y moral. No obstante, la voz que irrumpe en la segunda parte del poema -antiheaneyiana a todas luces- muestra el peligro de la instrumentalización demagógica de lo poético. La diferencia entre poner la balanza y el alambique en las manos de un poeta o en las de un partisano. Formas antagónicas de entender y sobrellevar un peso, de aliviar y poner remedio al mal de Atlas. ${ }^{13}$

\footnotetext{
13 En The Redress of Poetry (1995), Heaney argumenta con la misma imaginería de la balanza acerca de las demandas del activismo político sobre la poesía (personificadas en las dos voces anteriores de "Weighing In" y "The Flight Path" 4): "For the activist, there is going to be no point in envisaging an order which is comprehensive of events but not in itself productive of new events. Engaged parties are not going to be grateful for a mere image -no matter how inventive or original- of the field of force of which they are a part. They will always want the redress of poetry to be an exercise of leverage on behalf of their point of view; they will require the entire weight of the thing to come down on their side of the scales" (2).
} 


\section{REFERENCIAS BIBLIOGRÁFICAS}

BACHELARD, G. 1994 (1947). La tierra y los ensueños de la voluntad. Trad. Beatriz Murillo Rosas. México, D. F.: Fondo de Cultura Económica.

Chevalier, J. y A. Gheerbrant. 1982 (1969). Dictionnaire des symboles: mythes, reves, coutumes, gestes, formes, figures, couleurs, nombres. Paris: Robert Laffont/ Jupiter. 1996 (1982). The Penguin Dictionary of Symbols. London: Penguin

Cirlot, J. E. 2001 (1958). Diccionario de símbolos. Madrid: Siruela.

Frazier, A. 2001. "Anger and nostalgia: Seamus Heaney and the ghost of the father". Éire-Ireland 36, 3-4: 7-38.

Collins, F. 1997. "Enactments of desire". The Gettysburg Review 10, 2: 325-343.

Heaney, S. 1966. Death of a Naturalist. London: Faber and Faber.

. 1972. Wintering Out. London: Faber and Faber. 1975. North. London: Faber and Faber.

Faber. 1980. Preoccupations: Selected Prose 1968-1978. London: Faber and . 1987. The Haw Lantern. London: Faber and Faber. . 1995. The Redress of Poetry: Oxford Lectures. London: Faber and Faber. . 1996. The Spirit Level. London: Faber and Faber. . 2000. El nivel. Trad. Pura López Colomé. Edición bilingüe. México. . 2004. Anything Can Happen: A Poem and Essay by Seamus Heaney with Translations in Support of Art for Amnesty. Dublin: Townhouse.

O'BRIEn, E. 2002. Seamus Heaney: Creating Irelands of the Mind. Dublin: The Liffey Press.

2003. Seamus Heaney: Searches for Answers. London/Dublin/Sterling, Virginia: Pluto Press.

Oxford English Dictionary. 2001. Oxford: Oxford University Press.

Osterwalder, H. 1997. “An equable achievement: Seamus Heaney’s The Spirit Level”. Irish Studies Review 20: 30-35. 